\title{
UTILIZAÇÃO DO INVERSOR DE FREQÜÊNCIA EM SISTEMAS DE IRRIGAÇÃO PARA CONTROLE DE VAZÃO ${ }^{1}$
}

\author{
Jacinto de Assunção Carvalho², Carlos Rogério de Mello³, Roberto Alves Braga Júnior², \\ Carlos Henrique Reinato ${ }^{4} \&$ Márcio José de Santana ${ }^{4}$
}

\begin{abstract}
RESUMO
Objetivou-se, com este trabalho, avaliar o uso de um inversor de freqüência de 25 cv na irrigação, considerando-se diferentes demandas de água como manejo. A avaliação consistiu de uma análise de custos e benefícios, ambos anuais. O custo foi calculado com base no fator de recuperação do capital, para taxas de juros de 6 e $12 \%$ e períodos de amortização de 5 e 15 anos. O benefício consistiu da economia de energia proporcionada pelo inversor de freqüência em relação aos procedimentos usuais de controle da vazão. Relacionaram-se os parâmetros econômicos e de manejo da irrigação, com o objetivo de se avaliar o equipamento para qualquer situação de projeto. Como exemplo de aplicação, analisaram-se duas situações de controle de vazão comparadas ao inversor de freqüência em um projeto de irrigação. Foram determinadas, com auxílio do inversor de freqüência, as características hidráulicas, em função da vazão de uma motobomba de $25 \mathrm{cv}$ sob várias rotações. Observou-se que o inversor de freqüência pode ser viável, devendo-se avaliar a redução de potência advinda do seu uso e o tempo de funcionamento da irrigação, analisando-se a melhor forma de amortização.
\end{abstract}

Palavras-chave: irrigação, inversor de freqüência, análise econômica, energia

\section{UTILIZATION OF A VARIABLE FREQUENCY DRIVE IN IRRIGATION SYSTEMS TO CONTROL DISCHARGE}

\begin{abstract}
This work aimed to evaluate the use of a variable frequency drive of $25 \mathrm{HP}$ in irrigation systems to manage different water demands. The evaluation was done analyzing annual costs and benefits. The cost was calculated using interest rates of 6 and $12 \%$ and capital return periods of 5 and 15 years. The benefit was the energy saving by use of the variable frequency drive in relation to usual procedures to control discharge. The economic and irrigation management parameters were compared in order to evaluate several design situations. As an example, two situations of discharge control were compared with the use of a variable frequency drive in an irrigation project, suggesting forms for evaluation of the equipment under irrigated conditions. With the help of variable frequency drive, hydraulic characteristics and discharge for a pump of $25 \mathrm{HP}$, under several frequencies, were determined. It was demonstrated that the equipment can be used in irrigation systems, although it needs to be evaluated for the power reduction due to its use and working so as to analyze the best form of repayment of investment cost.
\end{abstract}

Key words: irrigation, variable frequency drive, economic analysis, energy

Recebido em 27/10/1999, Protocolo 123/99

${ }^{1}$ Extraído da dissertação de Mestrado do segundo autor, apresentada à UFLA

${ }^{2}$ Prof. do Departamento de Engenharia - UFLA, CP 37, CEP 37200 - 000. Fone: (0xx35) 829 1489. E-mail: jacinto@ufla.br

${ }^{3}$ M.Sc. em Engenharia Agrícola, Irrigação e Drenagem, Departamento de Engenharia UFLA. Fone: (0xx35) 821 7143. E-mail: crmello@ufla.br

${ }^{4}$ Acadêmicos do curso de Engenharia Agrícola da UFLA, Departamento de Engenharia. Fone: (0xx35) 8291489 


\section{INTRODUÇÃO}

O aumento de produtividade da agricultura é um dos maiores desafios do Brasil para o próximo século. A necessidade de aumentar a produção de alimentos advém do crescimento populacional e da necessidade de exportação, haja vista que a manutenção de uma balança comercial favorável é primordial ao desenvolvimento.

Para aumentar a produtividade, é necessário o empresário empregar toda tecnologia possível e, na agricultura, é essencial a aplicação correta de água às culturas, pois o seu desenvolvimento está atrelado a esta condição. A irrigação destaca-se como uma das principais técnicas a serem implantadas, porém grande parte das áreas irrigadas do país trabalha com energia elétrica proveniente do sistema interligado de geração e transmissão, ou com pequenas gerações locais, de origem hidráulica (Arruda, 1988).

O consumo de energia elétrica em sistemas de irrigação está concentrado na instalação de bombeamento, cujo bom desempenho reflete nas características hidráulicas e energéticas da irrigação, proporcionando os parâmetros hidráulicos projetados e consumo otimizado de energia (Scallopi, 1985).

O desenvolvimento de uma planta está intimamente relacionado à variação das necessidades hídricas ao longo do seu ciclo, conforme os estádios fenológicos atingidos pela mesma (Doorenbos \& Kassam, 1994).

O projeto de um sistema de irrigação deve ser desenvolvido para atender às necessidades da situação mais crítica (Bernardo, 1989; Olitta, 1989). Do ponto de vista do volume de água necessário, a maior quantidade deve ser aplicada ao estágio de desenvolvimento, que corresponde à fase de florescimento e enchimento dos frutos, para a maioria das culturas. Para as demais etapas, os volumes a serem aplicados são menores (Doorenbos \& Kassam, 1994).

Normalmente, maneja-se o volume de água necessário de duas formas. Bernardo (1989) e Azevedo Netto \& Alvarez (1991) citam o controle de vazão através do fechamento de registro na saída das motobombas, como sendo uma das práticas mais comuns; neste caso, há introdução de perda de carga acidental na curva do sistema, proporcionando desperdício de energia.

$\mathrm{O}$ outro manejo consiste em se trabalhar com tempos menores de irrigação, aplicando-se a maior vazão, o que proporciona a aplicação de volumes menores, não introduzindo perda de carga (Olitta, 1989).

Conzett \& Robechek (1983) citam que uma das formas de se manejar adequadamente um sistema de bombeamento visando reduzir vazão, é alterar a rotação da bomba até a obtenção da vazão necessária, de acordo com as leis de Rateaux; isto não implica em introdução de perda de carga e o tempo de funcionamento não é alterado. Tiago Filho (1996) cita que um equipamento capaz de produzir este efeito com rapidez e eficiência é o inversor de freqüência, que trabalha alterando a freqüência da tensão aplicada ao motor, possibilitando controlar a sua rotação; ainda segundo este mesmo autor, o uso do inversor de freqüência na indústria em sistemas de bombeamento com este objetivo é uma prática bastante comum, pois sua aplicabilidade econômica é justificável pela redução do consumo energético, em comparação com outros procedimentos, especialmente de fechamento de válvula.
Hanson et al. (1996) aplicaram o inversor de freqüência em alguns sistemas de irrigação com este objetivo, nos EUA, obtendo resultados satisfatórios, uma vez que a redução da potência consumida, provocada pelo uso do equipamento, gerou substancial economia de energia; isto permite especular sobre a viabilidade do mesmo para as condições brasileiras.

Atualmente, a análise da viabilidade de equipamentos não consiste apenas de caracterização técnica, devendo-se ter, necessariamente, uma avaliação econômica que considere as peculiaridades de cada caso, confrontando benefícios e custos, preferencialmente em âmbito anual (Coelho, 1979; Frizzone et al., 1994).

Dentro do exposto pretende-se, com este trabalho, avaliar a viabilidade do equipamento inversor de freqüência em instalação de bombeamento, visando ao controle de vazão, considerando-se características de manejo em sistemas de irrigação.

\section{MATERIAL E MÉTODOS}

\section{Caracterização hidráulica da motobomba utilizada, sob diversas rotações}

Trabalhou-se com uma motobomba trifásica de $25 \mathrm{cv}$, controlada por um inversor freqüência e conectada a um circuito hidráulico com retorno para o tanque de sucção. Primeiramente, determinaram-se as curvas características correspondentes a diferentes rotações, sendo que o inversor de freqüência promoveu as mudanças na rotação, colocando a bomba em valores que variaram de 3600 a $3000 \mathrm{rpm}$, a intervalos de 200 rpm. Para as determinações, trabalhou-se com valores de vazão variando de zero ("shut off") até aproximadamente $55 \mathrm{~m}^{3} \mathrm{~h}^{-1}$, a intervalos próximo de $8 \mathrm{~m}^{3} \mathrm{~h}^{-1}$, controlando-se a vazão através de um registro de gaveta na saída da bomba. As vazões foram medidas diretamente, através de um medidor eletrônico adequadamente instalado no circuito hidráulico.

Para cada vazão, calculou-se a altura manométrica fornecida pela bomba, determinando-se a pressão de recalque, através de um manômetro de Bourdon, e a pressão de sucção, com um vacuômetro. A altura manométrica total foi dada pela soma das leituras desses dispositivos (Carvalho, 1989). Com os dados de vazão e altura manométrica, calculou-se a respectiva potência hidráulica, conforme Porto (1998).

Foram feitas leituras da potência elétrica ativa consumida pelo motor, correspondente a cada carga submetida ao mesmo (vazão) diretamente no inversor de frequiência, através de wattímetro e, simultaneamente, com o intuito de monitorar adequadamente os valores, através de registros de leituras de tensão, corrente e fator de potência para cada vazão, em um medidor eletrônico microcomputadorizado, instalado pela CEMIG (Companhia Energética de Minas Gerais). A partir daí, calculou-se a potência elétrica ativa, conforme Silva et al. (1997).

Relacionando-se potência hidráulica e potência elétrica, obteve-se o rendimento global, ou seja, do conjunto motobomba. A caracterização hidráulica da bomba foi feita dispondo-se de dados, relacionando-se altura manométrica e vazão, rendimento global e vazão, e potência elétrica e vazão. Desenvolveram-se, conforme Yanagi Jr. et al. (1997) polinômios de terceiro grau para ajustar altura manométrica e vazão (Q) e rendimento global e vazão, uma vez que esses autores constataram significâncias estatísticas entre as grandezas e o polinômio cúbico. A potência 
ativa em função da vazão foi ajustada através de um polinômio quadrático, conforme sugerem Arens \& Porto (1989). Na Tabela 1 (A, B e C) constam os polinômios desenvolvidos para a motobomba.

Tabela 1. Curvas características ajustadas com seus respectivos coeficientes de determinação

\begin{tabular}{lll}
\hline \multicolumn{1}{c}{ Rotação } & \multicolumn{1}{c}{ Polinômios Ajustados } & $\mathrm{R}^{2}(\%)$ \\
\hline A. Altura Manométrica (mca) & \\
3600 & $81,5+0,1535 \mathrm{Q}-0,008866 \mathrm{Q}^{2}+9,371 \times 10^{-5} \mathrm{Q}^{3}$ & 96,4 \\
3400 & $70,2+0,2176 \mathrm{Q}-0,01354 \mathrm{Q}^{2}+1,4046 \times 10^{-4} \mathrm{Q}^{3}$ & 91,3 \\
3200 & $57,03+0,002165 \mathrm{Q}+3,31 \times 10^{-3} \mathrm{Q}^{2}-1,446 \times 10^{-4} \mathrm{Q}^{3}$ & 98,3 \\
3000 & $50,1+0,1989 \mathrm{Q}-0,006022 \mathrm{Q}^{2}-2,334 \times 10^{-5} \mathrm{Q}^{3}$ & 89,4 \\
\hline B. Rendimento Global (decimal) & \\
3600 & $0,00205+0,0294 \mathrm{Q}-5,07 \times 10^{-4} \mathrm{Q}^{2}+3,279 \times 10^{-6} \mathrm{Q}^{3}$ & 99,7 \\
3400 & $0,001937+0,0314 \mathrm{Q}-0,000571 \mathrm{Q}^{2}+2,836 \times 10^{-6} \mathrm{Q}^{3}$ & 99,1 \\
3200 & $0,00086+0,0291 \mathrm{Q}-0,000511 \mathrm{Q}^{2}+2,671 \times 10^{-6} \mathrm{Q}^{3}$ & 99,8 \\
3000 & $-0,00224+0,037 \mathrm{Q}-8,535 \times 10^{-4} \mathrm{Q}^{2}+6,983 \times 10^{-6} \mathrm{Q}^{3}$ & 99,8 \\
\hline C. Potência Elétrica (W) & 99,6 \\
3600 & $6,738,78+203,63 \mathrm{Q}+0,05375 \mathrm{Q}^{2}$ & 99,6 \\
3400 & $5917,91+133,64 \mathrm{Q}+1,269 \mathrm{Q}^{2}$ & 99,3 \\
3200 & $5237,02+115,32 \mathrm{Q}+0,7821 \mathrm{Q}^{2}$ & 96,7 \\
3000 & $4464,08+51,48 \mathrm{Q}+1,521 \mathrm{Q}^{2}$ & \\
\hline
\end{tabular}

\section{Aspectos considerados na análise econômica}

A análise econômica foi realizada conforme Frizzone et al. (1994) e Hanson et al. (1996) que sugerem a aplicação da teoria de custos para avaliação de retornos econômicos de equipamentos.

A avaliação consiste da comparação entre os benefícios e os custos, ambos anuais. Os benefícios foram calculados a partir da redução de energia consumida devido ao uso do inversor de freqüência ao longo do ano; este cálculo consiste da determinação da redução de potência produzida pelo equipamento em relação a outros procedimentos de controle de vazão, multiplicando-a pelo tempo de funcionamento durante o ano e pelo preço da energia, que no caso foi de $\mathrm{R} \$ 0,09050 \mathrm{kWh}^{-1}$ para tarifa convencional, uma vez que a potência instalada está abaixo de $50 \mathrm{~kW}$ considerando-se, ainda, o ICMS para o meio rural no Estado de Minas Gerais, conforme Comitê de Distribuição de Energia Elétrica (1994).

Os custos anuais consistem da anualização do capital investido, feita de acordo com Frizzone et al. (1994) e Hanson et al. (1996) pelo fator de recuperação do capital, que leva em consideração a taxa anual de juros e períodos de retorno do capital, através das equações abaixo:

$$
\begin{aligned}
\mathrm{FRC} & =\frac{(1+\mathrm{i})^{\mathrm{n}} \cdot \mathrm{i}}{(1+\mathrm{i})^{\mathrm{n}}-1} \\
\mathrm{CA} & =\mathrm{FRC} \cdot \mathrm{CE}
\end{aligned}
$$

em que FRC é o fator de recuperação do capital (decimal), i é a taxa de juros (decimal), n é período de amortização (anos), CA é o custo anualizado ( $\left.\mathrm{R} \$ \mathrm{ano}^{-1}\right)$ e $\mathrm{CE}$ é o custo do equipamento $(\mathrm{R} \$)$.

Trabalhou-se com taxas de juros de 6 e $12 \%$ ao ano e períodos de retorno do capital de 5 e 15 anos. O preço atual de um inversor de freqüência de $25 \mathrm{cv}$, conforme fabricante, é de $\mathrm{R} \$ 6689,00$, incluindo-se o IPI (Imposto sobre Produtos Industrializados).

Consideraram-se duas situações para aquisição do equipamento: 1) através de financiamento, gerando-se anuidades, sob dada taxa de juros, suficientes para pagamento no tempo estipulado pelo contrato que, normalmente, está em torno de 5 anos; 2) aquisição sem necessidade de empréstimo, podendo-se amortizar ao longo de toda a vida útil do equipamento que, conforme fabricante, é de 15 anos. Nesta última situação os retornos deverão ser superiores àqueles gerados por aplicações financeiras compatíveis com o valor do equipamento, como caderneta de poupança, que atualmente possui taxas anuais de $6 \%$.

Para análise da viabilidade, foi gerada uma tabela, relacionando-se redução de potência, tempos anuais de funcionamento, taxas de juros, períodos de amortização e um parâmetro que representa o percentual do valor do equipamento que pode ser obtido como retorno. Este parâmetro foi calculado dividindo-se o retorno anual (benefício menos custo) pelo preço do equipamento. Com esta tabela é possível avaliar-se qualquer situação de emprego deste inversor de freqüência, independentemente do projeto, bastando conhecer-se a redução de potência gerada pelo equipamento e o tempo de funcionamento anual do sistema de bombeamento. Estas análises foram feitas quando o câmbio era de US\$1,00=R $\$ 1,85$.

\section{Exemplo de aplicação}

Foi idealizado um projeto de irrigação visando ao controle de vazão, considerando-se que o sistema de bombeamento trabalha na sua condição ótima de rendimento, o que ocorre para uma vazão próximo de $40 \mathrm{~m}^{3} \mathrm{~h}^{-1}$. Considerou-se uma área irrigada de 18 ha, com desnível de 43,1 m em relação à captação, para irrigação por inundação de 6 tabuleiros de 3 ha cada um. A jornada de trabalho é de 15 horas diárias e a distância da área à captação é de $600 \mathrm{~m}$.

Escolheu-se uma tubulação de aço zincado dimensionada pelo critério de velocidades econômicas, conforme Bernardo (1989) encontrando-se um diâmetro nominal de 89 mm. A rugosidade absoluta, sugerida por Azevedo Netto \& Alvarez (1991) para este material, é de $0,125 \mathrm{~mm}$. O cálculo da curva da tubulação para atender à maior demanda (3ํo estágio) foi realizado conforme Mello \& Yanagi Jr. (1999) chegando-se à seguinte forma:

$$
\mathrm{H}_{\text {man }}=43,1+0,02051 \mathrm{Q}^{2}
$$

em que $\mathrm{H}_{\text {man }}$ é a altura manométrica em mca e Q, a vazão em $\mathrm{m}^{3} \mathrm{~h}^{-1}$. $\mathrm{Na}$ Tabela 2 constam os parâmetros considerados para determinação das vazões necessárias a cada estágio da cultura.

Tabela 2. Representação dos parâmetros agronômicos utilizados no projeto

\begin{tabular}{lccccccc}
\hline Estágio & Kc & $\begin{array}{c}\text { ETo } \\
\left(\mathrm{mm} \mathrm{dia}^{-1}\right)\end{array}$ & $\begin{array}{c}\text { Duração } \\
(\mathrm{dias})\end{array}$ & $\begin{array}{c}\text { Necessidade } \\
\left(\mathrm{m}^{3} \mathrm{dia}^{-1}\right)\end{array}$ & $\begin{array}{c}\mathrm{Q} \\
\left(\mathrm{m}^{3} \mathrm{~h}^{-1}\right)\end{array}$ & $\begin{array}{c}\text { Tempo Total } \\
(\mathrm{h})\end{array}$ \\
\hline I & 0,60 & 2,78 & 1,67 & 35 & 300 & 20,0 & 525 \\
II & 0,80 & 2,69 & 2,15 & 40 & 387 & 25,8 & 600 \\
III & 1,05 & 3,33 & 3,50 & 50 & 630 & 42,0 & 750 \\
IV & 0,85 & 3,35 & 2,85 & 35 & 513 & 34,2 & 525 \\
\hline
\end{tabular}

As comparações a serem feitas consideram os seguintes procedimentos de variação do volume de água:

Procedimento 1 - Corresponde ao manejo em que a bomba trabalha a 3600 rpm e a vazão demandada em cada estágio é controlada por registro, introduzindo perda de carga acidental. Os pontos de trabalho A, B, C e D, na Figura 1, gerados por este procedimento, fornecem a vazão necessária a cada estágio da cultura: 
Tubulação 1 (ponto D, 3ํo estágio) $\Rightarrow \mathrm{H}_{\operatorname{man}}=43,1+0,02051 \mathrm{Q}^{2}$ Tubulação 2 (ponto C, 4 estágio) $\Rightarrow \mathrm{H}_{\operatorname{man}}=43,1+0,031634 \mathrm{Q}^{2}$ Tubulação 3 (ponto B, 2 estágio) $\Rightarrow \mathrm{H}_{\operatorname{man}}=43,1+0,05709 \mathrm{Q}^{2}$ Tubulação 4 (ponto A, 1ํo estágio) $\Rightarrow \mathrm{H}_{\operatorname{man}}=43,1+0,09667 \mathrm{Q}^{2}$

Assim, Tubulação 1 diz respeito à equação do sistema correspondente ao estágio de maior demanda hídrica, sendo a equação de "projeto"; as demais Tubulações são equações originadas a partir da Tubulação 1, por fechamento de válvula (perda de carga) gerando os pontos de trabalho (A, B, C e D) que fornecem as vazões necessárias aos demais estágios mantendo-se, assim, a condição operacional do projeto, que é de $15 \mathrm{~h}$.

A Figura 1 mostra esta situação de trabalho.

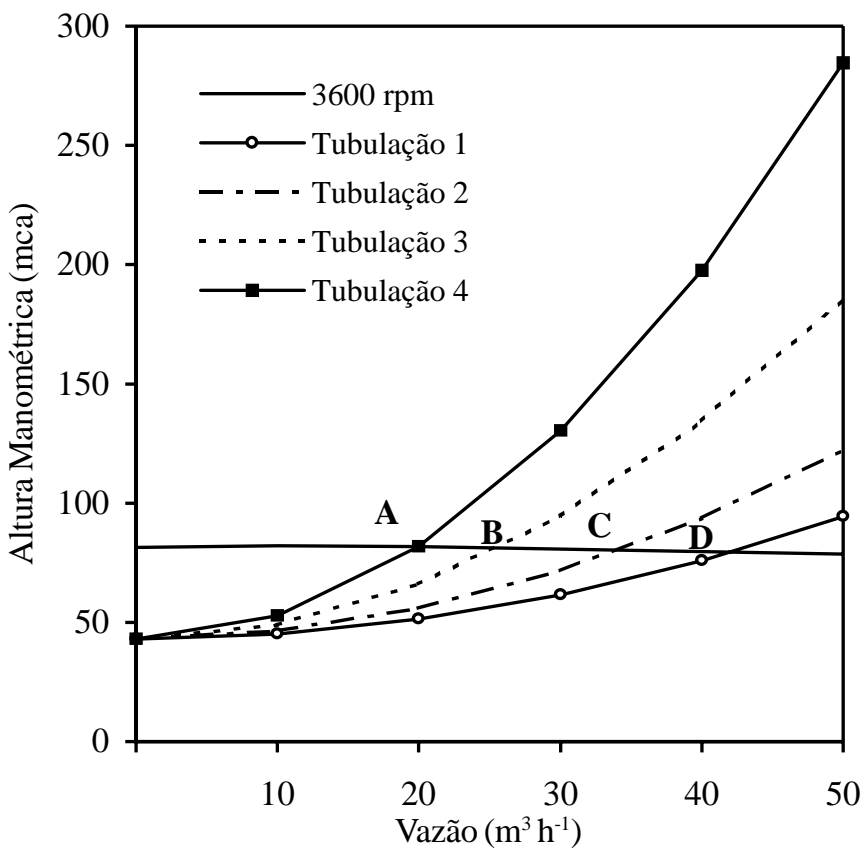

Figura 1. Pontos de trabalho da bomba (A, B, C e D) sob fechamento de válvula para controle de vazão

A potência consumida em cada ponto foi obtida com o polinômio correspondente à rotação de $3600 \mathrm{rpm}$, na Tabela $1 \mathrm{C}$.

Como os estágios da cultura têm duração diferente, considerou-se a economia de energia no ciclo como sendo a média das reduções de potência provocadas pelo inversor de freqüência em cada estágio, ponderando-as pela duração do respectivo estágio. Esta média corresponderá ao valor médio economizado durante o tempo de uso do inversor de freqüência.

Procedimento 2 - Este procedimento diz respeito ao manejo em que se trabalha com a vazão de projeto, porém aplicando-a em tempos menores, a fim de se obter o volume necessário ao estágio em que se encontra a cultura.

Trabalha-se com um percentual do tempo projetado para o estágio de maior vazão. Este percentual é calculado dividindose a vazão necessária a cada estágio pela vazão máxima de projeto (vazão necessária ao 3ำ estágio). A energia consumida nos demais estágios corresponde à multiplicação da potência necessária ao 3ํ estágio por tempos menores, em que para o $1^{\circ}$ estágio multiplica-se por $0,48 \mathrm{~h}$ para o 2o estágio, 0,61 h e para o 4ํe estágio,
0,81 h; a diferença entre esta energia consumida e a consumida utilizando-se inversor de freqüência, corresponde à economia obtida no estágio em $1 \mathrm{~h}$. A economia de energia foi calculada da mesma forma que no procedimento 1 , ou seja, mediante média ponderada pela duração do estágio.

Procedimento com inversor de freqüência - Pela Figura 2 observam-se os pontos de trabalho da bomba, sob variação de rotação com a curva da Tubulação 1 . Cada ponto de trabalho $(\mathrm{A}, \mathrm{B}, \mathrm{C}$ e D) fornece uma vazão correspondente à necessária em cada estágio, mantendo o manejo na condição operacional projetada, ou seja, de $15 \mathrm{~h}$ diárias de funcionamento, facilitando o manejo do sistema de irrigação.

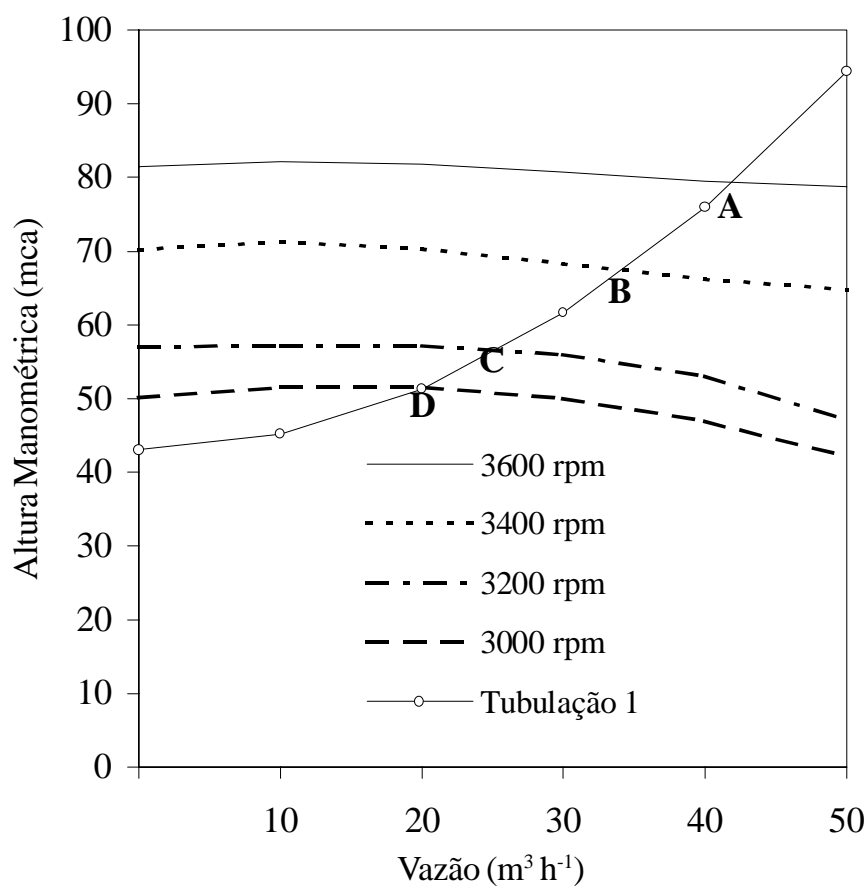

Figura 2. Representação dos pontos de trabalho (A, B, C e D) para controle de vazão, utilizando-se inversor de frequiência

A potência consumida por esta situação, em cada ponto de trabalho, pode ser obtida utilizando-se os polinômios da Tabela $1 \mathrm{C}$, que relacionam potência ativa e vazão.

\section{RESULTADOS E DISCUSSÃO}

\section{Discussão geral}

Através da Tabela 3 é possível se comparar várias situações de retorno associadas a taxas de juros, períodos de pagamento do capital (amortização), redução de potência e tempos de funcionamento, permitindo-se avaliar a viabilidade do inversor de freqüência de $25 \mathrm{cv}$.

Tomando-se como referência 5 anos para pagamento do capital investido, o sistema deverá funcionar no mínimo $2000 \mathrm{~h} \mathrm{ano}^{-1}$ e o inversor de freqüência proporcionará redução total de potência acima de $12 \mathrm{cv}$, para haver retorno positivo, considerando-se taxa de juros de $12 \%$ ao ano, dificultando o uso do equipamento.

Para esta situação, as anuidades são mais elevadas, uma vez que se dispõe de 5 anos para pagamento do capital; assim, é preciso que haja reduções de potência e tempo de funcionamento 
Tabela 3. Retorno, em termos percentuais, sobre o preço do inversor de frequiência de $25 \mathrm{cv}$ em função do número de horas de funcionamento ao ano, taxas de juros $(\mathrm{A}-6 \%$; $\mathrm{B}-12 \%)$, redução de potência e períodos de amortização $(\mathrm{N})$

\begin{tabular}{|c|c|c|c|c|c|c|c|c|c|c|}
\hline \multirow{3}{*}{$\Delta \mathrm{P}^{*}(\mathrm{cv})$} & \multicolumn{5}{|c|}{$\mathrm{N}=5$ anos } & \multicolumn{5}{|c|}{$\mathrm{N}=15$ anos } \\
\hline & \multicolumn{5}{|c|}{$\mathrm{h}$ ano $^{-1}$} & \multicolumn{5}{|c|}{$\mathrm{h}$ ano $^{-1}$} \\
\hline & 500 & 800 & 1500 & 2000 & 3500 & 500 & 800 & 1500 & 2000 & 3500 \\
\hline \multicolumn{11}{|l|}{ A. $6 \%$} \\
\hline 0,5 & $-23,4$ & $-23,3$ & $-22,8$ & $-22,5$ & $-21,6$ & $-10,0$ & $-9,8$ & $-9,4$ & $-9,1$ & $-8,2$ \\
\hline 3 & $-21,9$ & $-20,8$ & $-18,3$ & $-16,5$ & $-11,0$ & $-8,5$ & $-7,4$ & $-4,8$ & $-3,0$ & 2,4 \\
\hline 7 & $-19,5$ & $-16,9$ & $-11,0$ & $-6,8$ & 6,0 & $-6,1$ & $-3,5$ & 2,4 & 6,7 & 19,4 \\
\hline 12 & $-16,5$ & $-12,1$ & $-1,9$ & 5,4 & 27,2 & $-3,0$ & 1,3 & 11,5 & 18,8 & 40,6 \\
\hline \multicolumn{11}{|l|}{ B. $12 \%$} \\
\hline 0,5 & $-27,4$ & $-27,3$ & $-26,8$ & $-26,5$ & $-25,6$ & $-14,0$ & $-14,2$ & $-13,8$ & $-13,5$ & $-12,6$ \\
\hline 3 & $-25,9$ & $-24,8$ & $-22,3$ & $-20,5$ & $-15,0$ & $-13,0$ & $-11,8$ & $-9,2$ & $-7,4$ & $-1,9$ \\
\hline 7 & $-23,5$ & $-20,9$ & $-15,0$ & $-10,8$ & 2,0 & $-10,0$ & $-7,9$ & $-1,9$ & 2,3 & 15,0 \\
\hline 12 & $-20,5$ & $-16,1$ & $-5,9$ & 1,4 & 23,2 & $-7,4$ & $-3,0$ & 7,1 & 14,4 & 36,3 \\
\hline
\end{tabular}

anuais relativamente altos para proporcionar benefícios superiores aos custos. Estas características, segundo Hanson et al. (1996) e Scallopi (1985) podem ser limitantes para aplicação a sistemas de irrigação; contudo, deve-se considerar que haveria pagamento do financiamento neste período e restariam 10 anos de uso do equipamento, sem custo.

Considerando-se 15 anos para pagamento e taxa de juros de $6 \%$, caracterizando uma situação em que o irrigante dispõe de capital, podendo-se amortizar o equipamento ao longo de toda sua vida útil, trabalhando $800 \mathrm{~h} \mathrm{ano}^{-1} \mathrm{com}$ redução total de potência acima de aproximadamente $12 \mathrm{cv}$, os retornos serão positivos. Considerando-se, ainda, $1500 \mathrm{~h}^{2} \mathrm{ano}^{-1}$ com reduções próximo de $10 \mathrm{cv}$, os retornos são positivos, independentemente da taxa de juros, sendo de aplicabilidade mais razoável à irrigação que a situação de 5 anos para pagamento. Avaliando-se, por exemplo, um tempo médio de funcionamento de $2000 \mathrm{~h}$ ao longo do ciclo da cultura, com reduções médias de potência de $7 \mathrm{cv}$, a economia de energia seria suficiente para retornos positivos, independentemente das taxas de juro, justificando o uso do equipamento, especialmente se se trabalhar com duas safras anuais. Observa-se, portanto, que, dispondo-se de 15 anos para pagamento, a aplicabilidade do inversor de freqüência em sistemas de irrigação será justificável.

De maneira geral, deve-se ter atenção especial para com os parâmetros tempo de funcionamento e redução de potência, pois ambos são limitantes para tomada de decisão e devem ser cuidadosamente avaliados. Hanson et al. (1996) concluíram que em irrigação o tempo de funcionamento é extremamente determinístico, em termos de consumo energético, devendo ser analisado com ponderação, fato este que também pode ser observado neste trabalho.

O parâmetro tempo de retorno do capital também deve ser bem avaliado, pois se nota grande diferença, em termos de retorno, quando se comparam 5 e 15 anos para pagamento do investimento. Em irrigação, pequenas diferenças de funcionamento anual podem inviabilizar o inversor de freqüência, já que alterar o planejamento do manejo é complicado, podendo tornar-se inviável.

\footnotetext{
Análise do exemplo de aplicação Comparação entre inversor de freqüência e procedimento de manejo 1 - A Tabela 4 mostra a redução de potência associada a tempos de funcionamento correspondentes a cada estágio de desenvolvimento da cultura, considerando-se o procedimento 1 de manejo da vazão (controle de vazão feito por registro).
}

Tabela 4. Reduções de potência* proporcionadas pelo inversor de freqüência em relação ao procedimento de fechamento de válvula

\begin{tabular}{lcrrrc}
\hline Estágio & $\mathrm{Q}$ & Pot. 1 & Pot. 2 & $\Delta \mathrm{P}$ & $\begin{array}{c}\text { Tempo total } \\
\mathrm{h}\end{array}$ \\
& $\mathrm{m}^{3} \mathrm{~h}^{-1}$ & & $\mathrm{cv}$ & & \\
\hline I & 20,0 & 8,30 & 14,74 & 6,44 & 525 \\
II & 25,8 & 11,88 & 16,36 & 4,48 & 600 \\
III & 42,0 & 20,93 & 20,93 & 0,00 & 750 \\
IV & 34,2 & 16,29 & 18,73 & 2,44 & 525 \\
\hline
\end{tabular}

* Pot. 1: Potência gerada pelo uso do inversor de freqüência; Pot. 2: Potência gerada pelo uso de válvula $\Delta \mathrm{P}$ : Redução de potência

Calculando-se a média da potência reduzida, ponderada pela duração de cada estágio, encontra-se uma redução de 4,45 cv em $1650 \mathrm{~h}$ de uso do inversor de freqüência, para uma safra. Como se consideraram duas safras ao ano, o tempo total de funcionamento do equipamento ao ano será de $3300 \mathrm{~h}$. Pela Tabela 3, observa-se que o inversor de frequiência não será viável para amortização em 5 anos mas, considerando-se 15 anos, será viável, independentemente da taxa de juros. Deve-se, portanto, adotar a menor taxa de juros anual possível.

Se o irrigante dispuser de capital para aquisição do equipamento e conseqüentemente, amortizá-lo ao longo de toda a sua vida útil, o inversor de frequiência será viável, obtendo-se retornos positivos consideráveis, independente da taxa de juros.

Comparação entre inversor de freqüiência e procedimento de manejo 2 - A Tabela 5 mostra a potência reduzida pelo inversor de frequiência em comparação com o procedimento 2 (alteração do tempo de funcionamento, trabalhando-se com a vazão máxima).

Tabela 5. Representação da redução de potência pelo uso do inversor de freqüência* em comparação com o procedimento de redução do tempo de bombeamento

\begin{tabular}{|c|c|c|c|c|c|c|c|c|}
\hline Estágio & $\overline{\mathrm{Q} 1}$ & $\overline{\mathrm{Q} 2}$ & $\begin{array}{l}\mathrm{E} 1 \\
\mathrm{~kW}\end{array}$ & E2 & $\begin{array}{c}\text { Percentagem } \\
\text { do tempo }\end{array}$ & $\mathrm{T} 1$ & $\begin{array}{c}\Delta \mathrm{E} \\
\mathrm{kWh}\end{array}$ & $\begin{array}{c}\text { Pot. reduzida } \\
\mathrm{cv}\end{array}$ \\
\hline I & 20,0 & 42,0 & 6,102 & 7,385 & 48 & 525 & 1,283 & 1,75 \\
\hline II & 25,8 & 42,0 & 8,733 & 9,385 & 61 & 600 & 0,652 & 0,90 \\
\hline III & 42,0 & 42,0 & 15,386 & 15,386 & 100 & 750 & 0,000 & 0,00 \\
\hline IV & 34,2 & 42,0 & 11,973 & 12,460 & 81 & 525 & 0,487 & 0,66 \\
\hline
\end{tabular}

* Q1: Vazão aplicada pelo procedimento com inversor de frequiência, em $\mathrm{m}^{3} \mathrm{~h}^{-1}$
Q2: Vazão aplicada pelo procedimento de variação do tempo de irrigação, $\mathrm{em} \mathrm{m}^{3} \mathrm{~h}^{-1}$

E1: Energia consumida em $1 \mathrm{~h}$, com inversor de frequiência

E2: Energia consumida em um percentual de tempo necessário para aplicar volume correspondente ao aplicado pelo uso de inversor de freqüência

T1: Tempo de trabalho com o inversor de frequência, em $\mathrm{h}$

$\Delta$ E: Redução da energia consumida em $1 \mathrm{~h}$ pelo uso do inversor de freqüência

Percentagem de tempo: Corresponde ao percentual de tempo trabalhado pelo sistema de bombeamento para aplicar o mesmo volume que o procedimento com inversor de freqüência à vazão Q2

Pot. Reduzida: Redução de potência proporcionada pelo inversor de freqüência em $1 \mathrm{~h}$

Da mesma forma, a média ponderada da redução de potência para a primeira safra foi de $1,1 \mathrm{cv}$ em $1650 \mathrm{~h}$ de funcionamento do inversor de freqüência. Com o tempo de redução de 3300 h, pela Tabela 3 o inversor de freqüência não será viável para qualquer período de amortização ou taxa de juros. Nota-se que, comparado ao procedimento 2 (não há introdução de perda de carga) o inversor de frequiência ainda proporcionou economia de energia para este exemplo, devido ao fato de que há redução do rendimento mecânico da instalação para vazões maiores, conforme sugerem Yanagi Jr. et al. (1997). Deve-se ressaltar que o inversor de freqüência pode, em algumas situações, gerar consumo energético superior ao procedimento 2 .

Esta inviabilidade ocorreu devido ao fato de que as reduções de potência obtidas foram muito baixas, mostrando que a melhor opção para este irrigante controlar a vazão ao longo do ciclo da 
cultura, é variar o tempo de aplicação. Esta pequena economia de energia se deu porque houve grandes reduções de vazão e, com isto, o tempo de funcionamento necessário às situações também ficou bastante reduzido, vindo a refletir no consumo energético, uma vez que este é função direta do tempo de funcionamento. $\mathrm{O}$ manejo econômico mais adequado inclui jornadas diárias de 7,2 h durante o $1^{\circ}$ estágio; 9,15 h para o $2^{\circ}$ estágio e de 12,15 h para o 4 o estágio. Deve-se deixar claro que o inversor de frequiência produziu economia de energia, mas esta economia, traduzida em retorno, não supera as anuidades necessárias para pagamento do equipamento.

Deve-se examinar cuidadosamente cada projeto, pois a redução de potência e o tempo de funcionamento da irrigação são extremamente importantes e determinam a aplicabilidade do inversor de frequiência. Cada projeto tem as suas peculiaridades, que definem a viabilidade do equipamento. Esses exemplos mostram como se proceder para avaliar o emprego do inversor de freqüência, considerando-se as características de manejo da vazão em uma irrigação.

\section{CONCLUSÕES}

1. O uso do inversor de freqüência visando controlar vazão, pode ser viável, especialmente em se dispondo da vida útil do mesmo, para amortização do investimento devendo-se, no entanto, avaliar cuidadosamente a redução de potência e o tempo de funcionamento da irrigação durante o ano.

2. Uma vez verificados a redução de potência e o tempo de funcionamento, deve-se estudar adequadamente a forma de aquisição do equipamento, pois o período de retorno do capital é decisivo para a adoção da tecnologia.

3. O irrigante deve analisar a possibilidade de controlar o volume necessário a cada estágio, utilizando o manejo de redução do tempo de bombeamento, com a vazão de projeto, pois este pode ser mais atrativo, do ponto vista econômico, que o uso de inversor de freqüência.

\section{AGRADECIMENTOS}

Os autores agradecem à FAPEMIG (Fundação de Amparo à Pesquisa de Minas Gerais) pelo suporte econômico desta pesquisa; à CAPES, pela concessão de bolsa ao segundo autor e ao CNPq, pela concessão de bolsa ao primeiro autor.

\section{REFERÊNCIAS BIBLIOGRÁFICAS}

ARENS, H.G.; PORTO, R. de M. Para o desempenho de bombas. Revista DAE, São Paulo, v. 49, n.154, p.11-14, jan./mar. 1989.
ARRUDA, M.A. Análise de viabilidade da irrigação e participação da energia na composição de custos. In: SEMINÁRIO NACIONAL DE ENERGIA PARA IRRIGAÇÃO, 1, 1988, Belo Horizonte. Anais .... Belo Horizonte: CEMIG, CODI, Ministério da Irrigação, 1988. p.235-243.

AZEVEDO NETTO, J.M.; ALVAREZ, G.A. Manual de hidráulica. 7. ed. São Paulo: Edgard Blücher, 1991. 335p.

BERNARDO, S. Manual de irrigação. 5. ed. Viçosa: UFV, 1989. 596p.

CARVALHO, D.F. Instalações elevatórias. Bombas. 4. ed. Belo Horizonte: FUMARC, 1989. 355p.

COELHO, S.T. Matemática financeira e análise de investimentos. São Paulo: Ed. Nacional, EDUSP, 1979. 279p.

COMITÊDEDISTRIBUIÇÃODEENERGIA ELÉTRICA. Tarifas horo-sazonais azul e verde: Manual de orientação ao consumidor. 2. ed. Rio de Janeiro: CODI, 1994. 26p.

CONZETT, J.C.; ROBECHEK, J.R. Acionamentos de velocidade variável aplicados a bombas centrífugas. São Paulo: Reliance Electric Co., p.1-8, julho 1983. (Tradução de RACCA, C., Reliance Elétrica Ltda)

DOORENBOS, J.; KASSAM, A.H. Efeito da água no rendimento das culturas. Campina Grande: UFPB, 1994. 306p.

FRIZZONE, J.A.; BOTREL, T.A.; FREITAS, H.A.C. Análise comparativa dos custos de irrigação por pivô-central, em cultura de feijão, utilizando energia elétrica e óleo diesel. Engenharia Rural, Piracicaba, v.5, n.1, p.34-53, 1994.

HANSON, B.; WEIGAND, C.; ORLOFF, S. Performance of electric irrigation pumping plants using variable frequency drives. Journal of Irrigation and Drainage Engineering, New York, v.122, n.3, p.179-182, 1996.

MELLO, C.R. de; YANAGI JR., T. Escolha de bombas centrífugas. Revista Ciência e Agrotecnologia, Lavras, n.29, p. 1-27, 1999. Boletim Agropecuário

OLITTA, A.F.L. Os métodos de irrigação. 1.ed. São Paulo: Nobel, 1989.267p.

PORTO, R.M. Hidráulica básica. São Carlos: EESC-USP, 1998. 540p.

SCALLOPI, E.J. Exigências de energia para irrigação. Irrigação e Tecnologia Moderna. Brasília, v.21, n.2, p.13-17, 1985.

SILVA, J.P.; VIEIRA, O.J.; BRAGA JR., R.A. Eletricidade na agropecuária: Qualidade e conservação. v.2. Lavras: UFLA/ FAEPE, 1997. 102p.

TIAGO FILHO, G.L. Uso de bombas com rotação variável. Itajubá: EFEI. 1996. 19p. Mimeografado.

YANAGI JR., T.; FERREIRA, E.; COLOMBO, A.; MELLO, C.R. de. Método matemático para a estimativa analítica das curvas de desempenho de bombas centrífugas. Revista Ciência e Agrotecnologia. Lavras, v.21, n.4, p.484-490, out/dez. 1997. 